\title{
Severe cholestatic hepatitis following cloxacillin treatment
}

\author{
Sorel Goland, Stephen DH Malnick, Raia Gratz, Edith Feldberg, David Geltner, \\ Zev M Sthoeger
}

\begin{abstract}
Summary
We describe a 77-year-old woman who developed fatigue, fever, a maculopapular rash and severe cholestatic hepatitis two weeks after receiving cloxacillin and ofloxacin.
\end{abstract}

Keywords: hepatitis; cloxacillin; ofloxacin

Semisynthetic penicillins are widely used antibiotics. Hepatotoxicity (mainly cholestatic) associated with these drugs is a rare, but well described phenomenon. ${ }^{12}$ Several series of cholestatic hepatitis have been reported after treatment with flucloxacillin, ${ }^{2-4}$ whereas only isolated cases related to cloxacillin have been described..$^{5-10}$ Cholestatic jaundice may develop up to seven weeks following cessation of treatment. ${ }^{3}$ It may present as a mild disorder or as severe, prolonged cholestatic hepatitis which may lead to a fatal outcome despite cessation of the drug. ${ }^{25}$ The optimal treatment (in addition to discontinuation of the drug) is unclear. We describe a patient who developed a severe and prolonged cholestatic hepatitis which we believe was related to cloxacillin treatment.

\section{Case report}

A previously healthy 77-year-old woman underwent an elective repair of her left hallux valgus under local anaesthesia. She subsequently developed an operative wound infection and was treated intravenously for a total of 14 days with cloxacillin $6 \mathrm{~g} /$ day and ofloxacin 400 $\mathrm{mg} /$ day. Two weeks after cessation of the treatment she complained of increasing weakness and a pruritic maculopapular rash. Physical examination was unremarkable except for a widespread maculopapular rash with signs of scratching. There was neither abdominal tenderness nor hepatosplenomegaly. Laboratory tests revealed a haemoglobin of $11.6 \mathrm{~g} / \mathrm{dl}$, white blood cells $4.7 \times 10^{9} / 1$, with $9.5 \%$ eosinophils (total eosinophils $423 / \mathrm{ml}$ ), and platelets $273 \times 10^{9} / 1$. Prothrombin time was normal. The erythrocyte sedimentation rate was $66 \mathrm{~mm} / \mathrm{h}$. Serum glucose, electrolytes and renal function tests were all normal. Total serum bilirubin was $221 \mu \mathrm{mol} / 1$ (direct $144 \mu \mathrm{mol} / \mathrm{l}$ ). There was an increase of serum transaminases: alanine transaminase $144 \mathrm{IU} / 1$ (normal <41) and aspartate transaminase $313 \mathrm{IU} / 1$ (normal <37)). The alkaline phosphatase was 394 IU/l (nor- mal 53-128) with $\gamma$-glutamyl transferase of 212 IU/1 (normal 11-49). Serum cholesterol was $381 \mathrm{mg} / \mathrm{dl}$. Repeated serological tests for hepatitis A, B and C, CMV, EBV and Q fever, as well as antinuclear, antimitochondrial and antismooth muscle auto-antibodies, were all negative. IgE level was $354 \mathrm{IU} / \mathrm{ml}$ (normal 90-110).

Abdominal ultrasound and computed tomography (CT) were both normal except for the presence of several small gallstones with no signs of inflammation or bile duct obstruction. The jaundice deepened reaching a bilirubin of $391 \mu \mathrm{mol} / 1$ (mostly direct). Liver biopsy showed a portal tract infiltrate of lymphocytes and eosinophils, prominent cholestasis in the hepatocytes and focal hepatocellular necrosis, compatible with an exudative type of druginduced cholestasis. Peripheral blood lymphocyte proliferation tests with both ofloxacin and cloxacillin were negative.

After one month of jaundice the patient's general condition declined and it was decided to give $40 \mathrm{mg}$ prednisone treatment with a presumptive diagnosis of drug-induced cholestasis. Within 10 days there was a dramatic response in her general condition and a decrease in the bilirubin and transaminases which later returned to normal. She subsequently received ofloxacin for a urinary tract infection from her family physician and no illeffects were noted. The patient remains well with no signs of any liver damage, at two years of follow-up.

\section{Discussion}

We describe a patient who developed a prolonged, severe cholestatic jaundice 14 days after completing treatment with cloxacillin and ofloxacin for a wound infection. The jaundice was associated with allergic features such as rash and eosinophilia and responded dramatically to steroid treatment. Penicillinaseresistant pencillins are widely used drugs. Hepatotoxicity associated with their usage is not a common complication. Most reports involve series of cases following treatment with flucloxacillin, ${ }^{125}$ with an incidence in Australia of between 1:12000 and 1:100 000 and in Sweden of between 1:11000 and 1:30000. ${ }^{25}$ Flucloxacillin-related hepatotoxity is predominantly of a cholestatic type, ${ }^{25}$ found mainly in females, with a mean age of 58 years. The mean period of time from initiation of treatment to disease was 22.6 days. $^{2}$ There are only a few 
cases of cholestatic hepatitis associated with cloxacillin, as in our patient. ${ }^{5-10}$

In addition to cloxacillin, our patient was treated with ofloxacin. Ofloxacin is an antibiotic of the quinolone group and reports of hepatotoxicity associated with its use are extremely rare. ${ }^{11}$ All the cases demonstrated only mild acute hepatitis without features of cholestasis, which was dominant in our case. Our patient received an inadvertent rechallenge with ofloxacin with no cholestasis or liver enzyme abnormality. Thus, we believe that the cholestatic hepatitis observed in our patient was related to cloxacillin rather than to ofloxacin.

The usual histological features of druginduced cholestasis are divided into two types: canalicular ('bland') and exudative. The former type is pathologically 'bland' due to changes in the membrane of the liver cells. ${ }^{12}$ The exudative type, is predominantly cholestatic with some features of hepatocellular necrosis and inflammation (cholangitis) and is clinically hypersensitivity-mediated, usually associated with rash, fever and eosinophilia, as in our patient.

The mechanism of the reaction to the cloxacillin is unclear. The nature of the liver biopsy, the clinical presentation with rash and eosinophilia, and the dramatic response to the steroid treatment suggest a hypersensitivity allergic mechanism, which is not dose-dependent. The

1 Strickter BHCH, Brour M, Wilson JHP. Antibiotics. Penicillins. In: Strickter BHCH, Drug-induced hepatic Penicillins. In: Strickter BHCH, Drug-induced hepatic
injury, 2nd edn. Amsterdam: Elsevier, 1992; pp 175-83. 2 injury, 2nd edn. Amsterdam: Elsevier, 1992; pp 175-83. VJ. Flucloxacillin injury. Liver 1994;14:225-9.

3 Turner IB, Eckstein RP, Riley JW, Lunzer MR. Prolonged hepatic cholestasis after flucloxacillin therapy. Med $\mathcal{F}$ Aust 1989;151:701-5.

4 Miros M, Kerlin P, Walker N, Harris O. Fluoxacillin induced delayed cholestatic hepatitis. Aust NZ $\mathcal{F}$ Med 1990; 20:251-3.

5 Olsson R, WB-E, Sand L, Zettergren L, Hultcrantz R, Myrhed $M$. Liver damage from fluoxacillin/cloxacillin and dicloxacillin. $\mathcal{F}$ Hepatol 1992;15:159-61.

6 Enat R, Pollack S, Ben-Arie Y, et al. Cholestatic jaundice caused due to cloxacillin: macrophage inhibition factor test in preventing rechallenge with hepatotoxic drugs. BMF
$1980 ; 1: 982-3$.

\section{Learning points}

- cloxacillin can cause a severe cholestatic jaundice

- the jaundice may appear several weeks after stopping taking the medication

- steroid therapy can produce a dramatic response

patient received a daily dose of $6 \mathrm{~g}$ of cloxacillin which is the maximum recommended dose in the British National Formulary for severe infections. In addition, the bioavailability of cloxacillin is lower than that of flucloxacillin. ${ }^{1} \mathrm{~A}$ previous report of cloxacillin-associated hepatitis demonstrated a positive macrophage inhibition test, ${ }^{6}$ although in our patient there was no peripheral blood mononuclear cell response to cloxacillin. We believe that it is more likely that the severe jaundice seen in our patient was an idiosyncratic hypersensitivity reaction and not a direct dose-dependent reaction.

There are scarce data regarding treatment. The majority of cases resolve spontaneously, although fatal cases have been reported. ${ }^{2-5}$ Although our patient responded dramatically to steroids, the role of steroid treatment is uncertain. We suggest that corticosteroid treatment should be considered in patients with severe, prolonged, drug-induced cholestatic hepatitis with features of hypersensitivity.

7 Aderka D, Livni E. Use of macrophage inhibition factor and mast-cell degranulation tests for diagnosis of cloxacillininduced cholestasis. $A m \mathcal{F}$ Gastroenterol 1986;81:1084-6.

8 Konikoff F, Alcalay J, Halevy J. Cloxacillin-induced jaundice. Am $\mathcal{f}$ Gastroenterol 1986;81:1082-3.

9 Pascual J. Cloxacillin-induced cholestasis in renal allograft patient with chronic hepatitis (letter). Am $f$ Gastroenterol patient with chrom

10 Lotric S, Leijko-Zupanc T, Jereb $M$. Cloxacillin-induced cholestasis (letter). Clin Infect Dis 1994; 5:981-2.

11 Domingo P. Norfloxacin-induced hepatotoxicity (letter). $\mathcal{f}$ Hepatol 1990;11:277-8.

12 Kaplowitz MD. Drug-induced hepatotoxicity. Ann Intern Med 1986;104:826-39. 